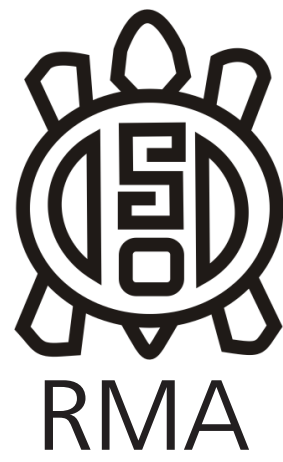

Dossier

\title{
El Tawantinsuyu y la minería lapidaria en el Noroeste argentino. Evidencias incaicas relacionadas con la explotación de turquesa en la cuenca de Ratones, Puna de Salta
}

\begin{abstract}
The Tawantinsuyu and lapidary mining in the North-Western Argentina. Inca evidence related to the exploitation of turquoise in the basin of

Ratones, Puna of Salta
\end{abstract}

Federico I. Coloca*

*CONICET, Instituto de Arqueología, Facultad de Filosofía y Letras, Universidad de Buenos Aires, Argentina. E-mail: fedeigco@hotmail.com

\begin{abstract}
Resumen
En este trabajo se analizan y discuten las evidencias arqueológicas vinculadas con la explotación lapidaria de turquesa provenientes de los sitios Cueva Inca Viejo y Abra de Minas, ubicados en la cuenca de Ratones, Puna de Salta. En recientes investigaciones se comprobó que el Cerro Inca Viejo constituye para el Noroeste argentino la primera fuente documentada con extracción prehispánica de dicho mineral. Si bien la extracción pudo haber comenzado en tiempos preincaicos, los incas habrían intensificado dicha actividad. Al respecto, se plantea que el Tawantinsuyu desarrolló en el área una organización para efectuar una minería eficiente a gran escala. Dicha organización implicó la instalación de un poblado como Abra de Minas y el establecimiento de áreas de trabajo cercanas a los pozos extractivos, como también la construcción de arquitectura ceremonial. En este sentido, se destaca la presencia de un registro vinculado con prácticas rituales que estaría relacionado con propiciaciones de festividades por parte del Imperio Inca, en donde se destacaba la hospitalidad y el agasajo, como parte de una estrategia asociada con una dominación simbólica sobre las poblaciones locales, y posiblemente sobre los grupos que fueron trasladados para trabajar en las minas.
\end{abstract}

Palabras clave: Minería lapidaria; Turquesa; Incas; Cuenca de Ratones; Puna de Salta.

\begin{abstract}
This work analizes and discusses the archaeological evidences linked to the lapidary exploitation of turquoise from the sites Cueva Inca Viejo and Abra de Minas, located in the basin of Ratones, Puna of Salta. Recent research has shown that Cerro Inca Viejo constitutes the first documented source of pre-Hispanic extraction of this mineral for the North-western Argentina. Although the extraction could have begun in pre-Inca times, the Incas would have intensified this activity. Likewise, it is stated that the Tawantinsuyu developed in the area an organization to carry out an efficient large-scale mining. This organization involved the installation of a settlement such as Abra de Minas and the establishment of work areas close to the extractive wells, as well as the construction of ceremonial architecture. In this sense, it is highlight the presence of registry linked to ritual practices it would be related to propitiations of festivities realized for the Inca Empire, in which hospitality and guested were highlighted, as part of a strategy associated with a symbolic domination over the populations local, and possibly on the groups that were transferred to work in the mines.
\end{abstract}

Keywords: Lapidary mining; Turquoise; Incas; Basin of Ratones; Puna of Salta.

Dentro de las estrategias humanas para el aprovisionamiento de recursos líticos, la minería lapidaria representa una explotación de escalas mayores, cuyas particularidades pueden observarse en los procesos de extracción, circulación y uso de la materia prima (García-Albarido, 2007). Este tipo de actividad, a su vez, tiene implicancias sociales que pueden vincularse con la organización de la explotación (e.g. necesidad de mano de obra, estructuración de áreas de trabajo, confección de instrumentos de trabajo, etc.) (García-Albarido, 2007). En los Andes Centro Sur, la actividad minera, aunque de manera escasa, se encuentra evidenciada en diferentes 
contextos prehispánicos tardíos (Angiorama y Becerra, 2014; Figueroa et al., 2013; González y Westfall, 2008; Núñez, 1999; Salazar, 2008; Westfall y González, 2010). Sin embargo, fue bajo el dominio del Imperio Inca que la explotación de minerales alcanzó su mayor escala (Berenguer y Salazar, 2017; González et al., 2017; Núñez, 1999; Salazar y Vilches, 2014; Salazar et al., 2013).

En términos generales, se ha propuesto que uno de los factores determinantes de la expansión del Tawantinsuyu hacia los Andes meridionales habría sido la extracción de minerales (Raffino, 1981). Para poder desarrollar estos objetivos, los colonizadores necesitaron proveerse tanto de mano de obra como también de un sistema que sostuviera dicha inversión. En este contexto, con el fin de mantener dicho sistema de manera más efectiva, el dominio estatal se basó en un manejo conjunto del control ideológico, militar y ceremonial (Raffino, 1981). Estas características han sido descriptas para diferentes sitios del Norte de Chile, en donde se ha planteado que a partir de la minería, y su simbología asociada, el Imperio reorganizó los espacios sociales y sagrados (Salazar, 2008; Salazar et al., 2013). Sin embargo, para el Noroeste argentino (NOA), este tipo de registro es aún escaso. A su vez, el desarrollo de actividades extractivas realizadas en épocas coloniales e históricas han dificultado enormemente el descubrimiento de actividades previas (Angiorama y Becerra, 2014).

En este trabajo se presentan evidencias arqueológicas vinculadas con actividad minera prehispánica en el NOA. Específicamente, el objetivo es caracterizar la explotación de turquesa en contextos de dominación Inca en la cuenca de Ratones, Puna de Salta (Figura 1). Para ello, se analiza y discute la diversidad arqueológica de los sitios Cueva Inca Viejo y Abra de Minas. En este sentido, se debe destacar que el cerro donde se ubica la Cueva Inca Viejo constituye la primera fuente documentada en el NOA con actividad prehispánica para la extracción de dicho mineral (López et al., 2018a; López et al., 2018b). La presencia de turquesa se comprobó a través de análisis de MEB-EDX y DRX, tanto de muestras extraídas de las paredes de la cueva como de rocas y cuentas recuperadas en contextos estratigráficos (López et al., 2018a). Esta nueva información toma relevancia si consideramos que hasta la actualidad, en la región de los Andes Centro Sur, la evidencia de explotación de minerales de cobre y piedras preciosas tales como la turquesa había sido solamente registrada en sitios del Norte de Chile, como Chuquicamata, Mina Las Turquesas y El Abra (González y Westfall, 2008; Salazar, 2008; Westfall y González, 2010).

A continuación, se presentan evidencias arqueológicas que permiten discutir sobre la organización y el funcionamiento que el Tawantinsuyu desarrolló en la cuenca de Ratones en torno a la extracción de turquesa en el Cerro Inca Viejo. Al respecto, se consideran distintos indicadores relacionados con cuestiones tecnológicas y económicas vinculadas con dicha actividad (e.g. técnicas extractivas, patrón de asentamiento, delimitación de áreas de trabajo, etc.), como también con diferentes aspectos sociales que pudieron estar implicados (e.g. utilización de mano de obra local, conquista ritual, comensalismo, etc.).

\section{El Tawantinsuyu y la explotación minera en la cuenca de Ratones.}

Cueva Inca Viejo es una cavidad rocosa ubicada a $4.312 \mathrm{msnm}$, sobre el faldeo del cerro homónimo que se erige al Este del salar de Ratones (Figura 1). En ella se reconocen diferentes indicadores vinculados con el proceso extractivo minero, como la apertura antrópica de socavones y galerías secundarias (Figura 2A), en cuyas paredes se observan marcas de piqueteado (Figura $2 \mathrm{~B}$ ) y relictos de vetas del mineral extraído (López et al., 2018a; 2018b).Asimismo, también se ha reconocido una intensa modificación antrópica relacionada con trabajos mineros a lo largo del cerro, principalmente vinculada con la apertura de pozos de grandes dimensiones (Figura 2C y D) (Coloca, 2017b; López et al., 2018b).Entre las evidencias artefactuales asociadas con la actividad minera
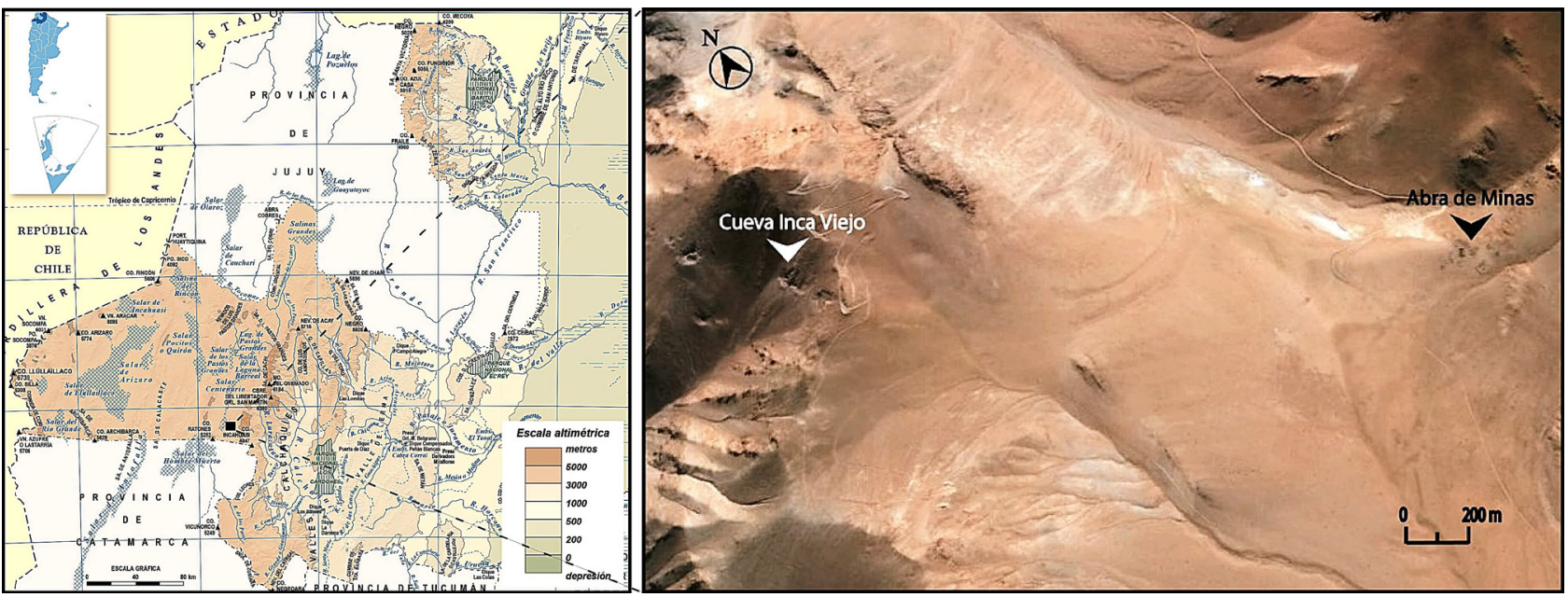

Figura 1. Ubicación de la cuenca de Ratones y los sitios Cueva Inca Viejo y Abra de Minas.

Figure 1. Location the basin of Ratones and Cuevas Inca Viejo and Abra de Minas sites. 
prehispánica, en el interior de la cueva se destaca el hallazgo de rocas $(\mathrm{N}=1996)$ y cuentas de turquesa $(\mathrm{N}=11)$ (Figura 2E); martillos líticos (Figura 2F), recuperados tanto en superficie $(\mathrm{N}=28)$ como en capa $(\mathrm{N}=1)$; y tiras de cuero (Figura 2G), presumiblemente utilizadas para enmangar dichos instrumentos (López et al., 2018a; 2018b). Se debe destacar que los martillos de piedra han sido señalados como uno de los elementos más confiables para determinar el trabajo minero en épocas prehispánicas (Salazar y Vilches, 2014). Los fechados radiocarbónicos del sitio demuestran ocupaciones humanas preincas (ca. 700 DC-1300 DC) e incas (ca. 1500 DC). Sin embargo, hasta el momento no se ha podido definir una estratigrafía con componentes definidos. Esta situación parecería relacionarse con la modificación producida por una intensa actividad inca, quienes habrían alterado el registro arqueológico de ocupaciones previas (para más información ver Coloca, 2017b; López et al.,2018b).

En cuanto a la construcción arquitectónica Inca en el sector del Cerro Inca Viejo, parecería haber sido dispuesta en base a una organización orientada a la explotación minera. En primer lugar, se encuentra la presencia de un camino, con un muro de contención, por el cual se accede a la cueva descendiendo desde uno de los faldeos del cerro. En diferentes puntos a lo largo de dicho camino, y sobre otros sectores del cerro, se registraron 13 recintos simples (Figura 2C), en su mayoría de planta cuadrangular, asociados con algún pozo o socavón (Figura 2D) (Coloca, 2017b). Entre ellos se destaca un recinto rectangular de $4,7 \times 12$ m, que en términos morfológicos puede definirse como una kallanka inca, el cual se encuentra cercano a un pozo de grandes dimensiones (30 m de diámetro). Si bien dichos recintos aún no han sido excavados, el material de superficie y su proximidad con los pozos extractivos hacen presumir que fueron destinados a alguna tarea relacionada con la minería. Al respecto, es importante resaltar en este sector la recurrencia en superficie de herramientas líticas vinculadas con esta actividad (e.g. yunques y martillos), tanto dentro de algunos de estos recintos como en sus proximidades. Asimismo, se registraron otras estructuras, localizadas en los puntos más altos del área, que habrían estado destinadas a otras funciones, relacionadas con las estrategias de control del acceso al cerro y la cueva por parte del Imperio. Desde ellas se puede observar el sitio Abra de Minas, el faldeo del cerro, y el camino que conduce a la cueva (Coloca, 2017b).

La presencia Imperial se evidencia a su vez en la cerámica de estilo Inca, aunque también se documenta un tipo negro pulido y escasos fragmentos de un tipo negro grabado, los cuales podrían corresponder a ocupaciones preincas (Coloca, 2017b). A su vez, en el sector Norte de la galería principal, se recuperó una envoltura de gramíneas que contenía plumas de diversas aves, huesos de camélidos, cerámica y restos vegetales. Este hallazgo ha sido interpretado como una ofrenda ritual, y ha sido fechado para contextos Incas (ver López et al. 2018b).

En lo referente al registro arqueológico de la cueva, no se puede soslayar la presencia de distintos indicadores asociados con el tráfico macrorregional y las prácticas rituales. Entre ellos, resalta la representación de pinturas rupestres con distintos motivos de caravaneo (antropomorfos guiando llamas alineadas y atadas). Dentro del material arqueológico se puede nombrar la presencia de obsidiana de diversas fuentes ${ }^{1}$, plumas de aves exóticas y semillas y frutos provenientes de valles mesotermales y yungas. Al respecto, es notable la evidencia de semillas de cebil, una planta alucinógena relacionada con prácticas rituales, como también de maíz y semillas de algarrobo, vinculadas con la producción de bebidas alcohólicas como la chicha y la aloja (López et al.,2015).

En relación con los indicadores asociados con prácticas ceremoniales, es importante destacar la presencia de una estructura compleja ubicada en el talud que se encuentra por delante de la boca de entrada de la cueva, y por encima de un sector aplanado antrópicamente. Esta construcción se compone de un muro superior de más de $2 \mathrm{~m}$ de alto, una plataforma de $1 \times 4 \mathrm{~m}$, una escalera de 19 escalones delimitado por muros dobles, y un recinto central, que en el sector inferior se encuentra delimitada por un muro simple de $10 \mathrm{~m}$ de largo (para más información ver López et al., 2018a; 2018b). Los atributos generales la permitieron caracterizar como una variedad de ushnu (López et al., 2018b).

Por su parte, Abra de Minas es un sitio a cielo abierto ubicado a $4.250 \mathrm{msnm}$, sobre una meseta cercana a una vega de altura. El sitio se encuentra a $2 \mathrm{~km}$ de Cueva Inca Viejo y se caracteriza por la presencia de 92 estructuras y una gran cantidad de material arqueológico superficial, principalmente cerámico (López y Coloca, 2015; Coloca, 2017b). Si bien los fechados radiocarbónicos se ubican dentro de un rango cronológico similar al de Cueva Inca Viejo, representando principalmente contextos preincas e incas (López et al., 2018b), Abra de Minas se destaca por una variedad de indicadores de una fuerte presencia estatal. Al respecto, la arquitectura responde, en su gran mayoría, a características propiamente imperiales, entre las que resaltan los Recintos Perimetrales Compuestos (RPC), una kallanka y una plataforma ceremonial en el sector de la vega (Coloca, 2017a). Esta última estructura se trataría de un ushnu, y se caracteriza por su planta cuadrangular y una alta inversión de energía en su construcción (Coloca, 2017b; López et al., 2018b). A partir del análisis arquitectónico, se ha planteado que el sitio pudo albergar una cantidad considerable de personas, posiblemente centenares (Coloca, 2017a; 2017b). A su vez, la diversidad en los tamaños y plantas de la arquitectura y su distribución diferencial dentro de la estructuración del asentamiento ha sido vinculada con la presencia de jerarquización. En este sentido, se ha señalado un conjunto de construcciones que detentan una posición privilegiada en cuanto a la circulación y al control visual de todo el poblado, las cuales fueron asociadas con ocupantes de mayor estatus, tal vez encargados de

\footnotetext{
1 En Cueva Inca Viejo se determinó la presencia de cuatro tipos de obsidianas: Tocomar, Quirón y Archibarca, de la provincia de Salta; y Ona y Zapaleri, de Jujuy (Coloca, 2017b).
} 
la dirección y organización del asentamiento. Dentro de dicha distribución, los RPC habrían sido conjuntos de residencia de individuos directamente relacionados con el Imperio, mientras que el resto de los recintos habitacionales pudieron haberse destinado para pobladores de menor jerarquía, posiblemente mitmaqkuna o mitayos locales (Coloca, 2017a). En relación con esta idea, en contextos similares, como por ejemplo en la Puna de Atacama, ha sido planteado que la mano de obra especializada que requería el trabajo minero, bajo el dominio del Tawantinsuyu, fue sustentada por medio del traslado de personas (Núñez et al., 2003).

Dentro del material artefactual de Abra de Minas predomina la cerámica Inca, con decoración geométrica (rombos, triángulos, reticulados, entre otros) y fitomorfa ("helechos") (Coloca, 2017b). A su vez, se reconocieron formas típicas estatales como aríbalos, platos (algunos de asas ornitomorfas) y ollas en pedestal (Coloca, 2017b). Estos tipos de vasijas han sido vinculados con actividades ceremoniales, posiblemente fiestas donde se compartieron y consumieron alimentos y bebidas, que habrían sido patrocinadas por el Estado (Bray, 2004; Williams et al., 2005). En este sentido, se puede destacar también la presencia de un mortero múltiple confeccionado sobre un bloque cercano a un RPC, seguramente utilizado para la preparación de bebidas y/o alimentos (López et al., 2018b).

Se debe resaltar que, por su ubicación, Abra de Minas detenta un punto estratégico para el control del tránsito hacia el Norte y el Sur, como también del acceso al cerro y a la Cueva Inca Viejo. En este sentido, el asentamiento se encuentra construido a la vera de un camino principal Inca, con sentido Norte-Sur, con rumbo hacia el oasis de Antofagasta de la Sierra, en la Puna de Catamarca. A su vez, rutas secundarias transversales conectarían el sitio con los valles y quebradas orientales y también con el Norte de Chile (Raffino, 1981).

En cuanto a las ocupaciones previas a la llegada del Imperio Inca en la cuenca de Ratones, se ha propuesto que el área habría conformado un lugar de importancia dentro de una red de intercambio interregional, en donde Abra de Minas sirvió como un paraje relevante en el tránsito caravanero (Coloca, 2017b). Asimismo, se sugiere que la relevancia de la Cueva Inca Viejo habría estado relacionada con la extracción de turquesa, la cual habría circulado hacia diferentes regiones dentro de la red caravanera. En este contexto, la evidencia parece señalar la importancia simbólico-ritual de este lugar desde tiempos preincas, en donde la cueva y el cerro, como fuente de mineral precioso, pudieron desempeñar un rol de importancia, posiblemente como wakas (e.g. Bouysse-Cassagne, 2005; Cruz, 2013). Posteriormente, la importancia minero-ritual de la Cueva Inca Viejo habría motivado el arribo del Imperio Inca en el área (Coloca, 2017b; López et al., 2018b).

\section{Discusión}

El registro arqueológico de la cuenca de Ratones

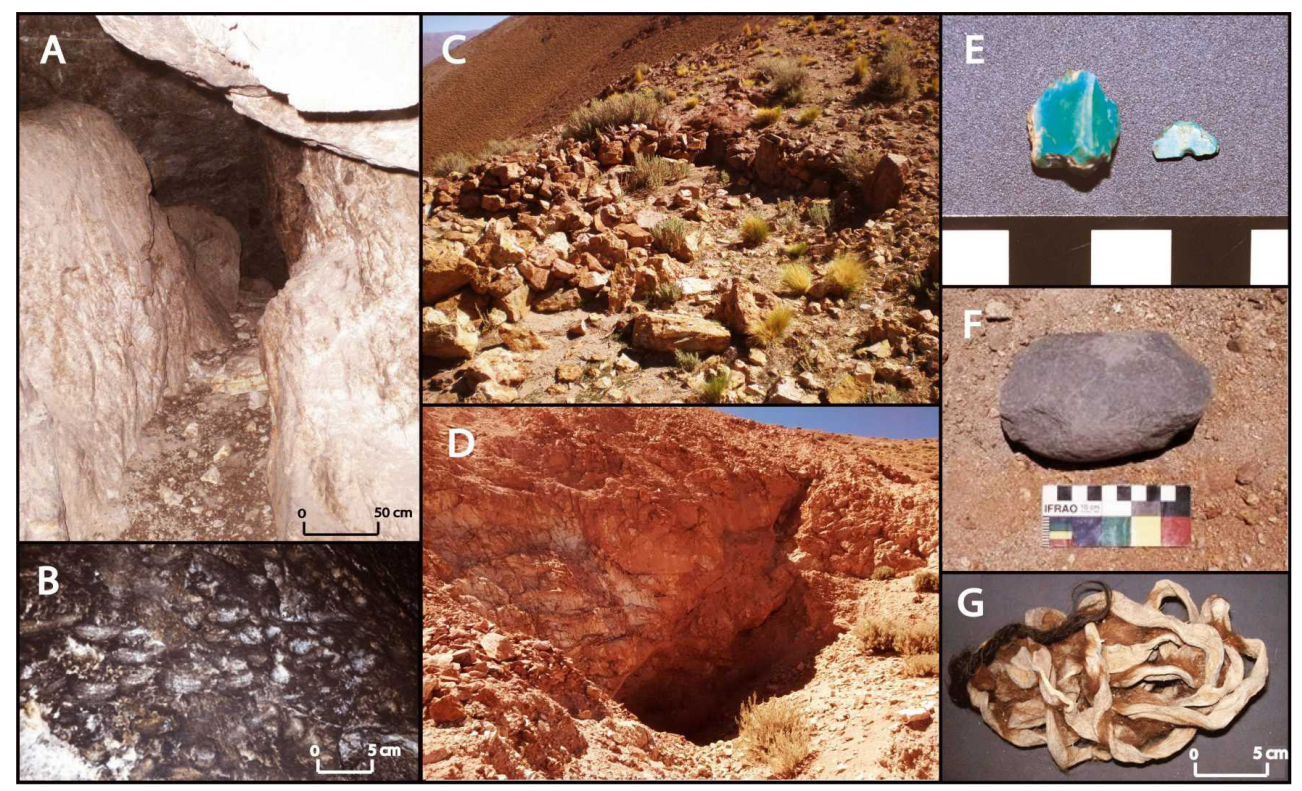

Figura 2. Indicadores de actividad minera en la cuenca de Ratones: A) galería antrópica al interior de Cueva Inca Viejo, B) Marcas de piqueteado sobre las paredes de las galerías de Cueva Inca Viejo, C) Estructura en el faldeo del Cerro Inca Viejo, D) Pozo minero en el Cerro Inca Viejo, E) Roca y cuenta fragmentada de turquesa, F) Martillo lítico superficial, G) Tiras de cuero, asociadas con el enmangue de martillos líticos.

Figure 2. Indicators of mining activity in basin of Ratones: A) Anthropic gallery inside Cueva Inca Viejo, B) Picket marks on walls of the galleries of Cueva Inca Viejo, C) Structure on slope of Cerro Inca Viejo, D) Mining well in Cerro Inca Viejo, E ) Turquoise rock and fragmented turquoise bead, F) Surface lithic hammer, G) Leather strips, associated with the hafting of stone hammers 
constituye un aporte para el estudio de la explotación minera y su relevancia en la expansión Inca en el NOA. Particularmente, la información obtenida permite discutir la importancia que el Tawantinsuyu le destinó a la extracción de la turquesa en el área. Por otra parte, no se ha podido avanzar demasiado sobre las prácticas

mineras de ocupaciones previas, ya que la evidencia se encuentra solapada por la intensa remoción llevada a cabo posteriormente por el Imperio. En este aspecto, se debe destacar la inversión que los Incas destinaron para el desarrollo de dicha actividad, como también las diferentes estrategias de control utilizadas, las cuales debieron conllevar sus implicancias sociopolíticas.

Las evidencias documentadas hasta el momento, como la apertura de galerías al interior de la cueva, los socavones y pozos expuestos a lo largo del cerro, y la presencia de martillos líticos, entre otros indicadores, permiten sostener que la Cueva Inca Viejo constituyó una mina lapidaria dedicada al trabajo de extracción. En este sentido, se reconocen las etapas iniciales de la secuencia de lapidación, relacionada con la obtención de los nódulos del mineral buscado (García-Albarido, 2007). Asimismo, el registro de una gran cantidad de rocas de turquesa, en su mayoría con relictos de la roca madre, podría asociarse con una selección y limpieza primaria de los nódulos de dicho mineral (sensu García-Albarido, 2007).

A su vez, todavía queda determinar si en el sector de Cueva Inca Viejo y/o en Abra de Minas fueron realizadas etapas posteriores dentro de la cadena operativa del trabajo lapidario. Contrariamente a la evidencia de otros sitios de características similares, como por ejemplo Mina Las Turquesas, en Chile (González et al., 2017), aun no se ha recuperado ninguna evidencia que pueda relacionarse con tareas como el chancado o la formatización (e.g. perforadores). Sin embargo, la presencia de cuentas confeccionadas con el mismo mineral que estaba siendo extraído del cerro, permiten plantear la hipótesis de una manufactura local. A su vez, futuras excavaciones de estructuras en las inmediaciones de los pozos extractivos, como también en diferentes recintos de Abra de Minas, podrían proporcionar nueva evidencia sobre el proceso lapidario llevado a cabo en la cuenca de Ratones.

Dentro de este contexto, se debe destacar la importancia que el Tawantinsuyu le destinó a la propiciación de festividades y ceremonias. En este sentido, la información recuperada hasta el momento en la cuenca de Ratones sugiere un escenario en donde el control ejercido podría estar asociado a su vez, con un aspecto de la dominación simbólica de la región por parte del Estado mediante la conquista de lugares sagrados, específicamente vincula dos con la mina y el cerro. En sitios mineros del Norte de Chile, esta apropiación habría incluido la instalación de enclaves estatales en cercanías de las minas para la residencia de colonos especializados (González et al., 2017). En el caso de la cuenca de Ratones Abra de Minas habría cumplido dicha función.
La relación existente entre sitios mineros incaicos y la realización de prácticas ceremoniales ha sido evidenciada en otros contextos andinos, en donde el Estado ejerció un control sobre los rituales asociados con las wakas mineras como forma de legitimar su poder sobre la producción (Salazar et al., 2013).De esta manera, el hallazgo de arquitectura ceremonial en Ratones puede considerarse un indicador del control ejercido por los Incas sobre la producción minera. Asimismo, el registro de artefactos suntuarios recuperados en las excavaciones de la cueva, como las plumas de diversos colores, el envoltorio de paja en forma de ofrenda, el cebil o las rocas y cuentas de mineral de turquesa, entre otras evidencias, podría asociarse también con este tipo de prácticas. Por lo tanto, se puede sostener que el Tawantinsuyu desarrolló, principalmente en el sector de la Cueva Inca Viejo, un espacio minero-ritual, como se ha expresado también para sitios del Norte de Chile (Berenguer y Salazar, 2017; Salazar et al., 2013, Soto y Salazar, 2016). Esta interpretación reforzaría la idea de un desarrollo de prácticas ceremoniales en la Cueva Inca Viejo de naturaleza más bien restringida y controlada por un determinado grupo de personas, que manejaría un tipo de materialidad suntuaria específica y limitada (Coloca,2017b).

Por otra parte, la plataforma de Abra de Minas puede vincularse con algún tipo de ceremonia de carácter relativamente abierto, en donde pudieron participar una gran cantidad de personas (Coloca, 2017b). Sumado a ello, la diversidad cerámica recuperada en el sitio avala la idea de festividades de hospitalidad brindadas por el Imperio, seguramente hacia los pobladores locales y/o mineros que habrían sido transportados hasta allí, en donde la característica común era el agasajo, por medio de la elaboración y distribución de comidas, y principalmente bebidas alcohólicas. Este tipo de prácticas fueron descriptas para diferentes sitios incaicos en el NOA, y han sido interpretadas como parte de una estrategia de dominación simbólica por parte del Tawantinsuyu (Giovannetti, 2015; Williams et al., 2005).

En relación con esta discusión, el hallazgo en Cueva Inca Viejo de maíz y semillas de algarrobo, a partir de las cuales se preparan la chicha y la aloja respectivamente, además de reforzar la idea de consumo de bebidas en contextos ceremoniales, demuestran que los Incas también proporcionaron también los bienes necesarios para dichos preparativos. Esta cuestión es informativa respecto de la organización bajo la cual se instaló el Imperio en el área, en donde estaba implicada la conexión con zonas que podían proporcionarle el abastecimiento requerido, no solo para dichas celebraciones, sino también para el mantenimiento de una instalación como Abra de Minas. De esta manera, se puede comprobar que el Tawantinsuyu mantuvo un eficiente sistema mediante el cual se suministraba de materiales y recursos, así también como de información y, seguramente, mano de obra destinada a la explotación minera.

Buenos Aires, 30 de abril de 2019. 


\section{Agradecimientos}

Al CONICET. A los compañeros/as de equipo y los que participaron de las campañas. Especialmente, agradezco a Fabiana Skarbun, Manuel Cueto y Ariel Frank por la invitación a participar de este dossier. A los evaluadores, cuyas observaciones y sugerencias permitieron mejorar el trabajo.

\section{Referencias Citadas}

Angiorama, C. y Becerra, M. (2014). "Como en ella jamás ha habido minas...". Minería y metalurgia en la puna de Jujuy durante momentos prehispánicos tardíos. Relaciones de la Sociedad Argentina de Antropología, XXXIV (2), 313-332.

Bouysse-Cassagne, T. (2005). Las minas del centro-sur andino, los cultos prehispánicos y los cultos cristianos. Bulletin de I'Institut Français d'Etudes Andines, 34(3), 443-462.

Berenguer, J. y Salazar, D. (2017). Territorialización del modelo minero inkaico en el río Salado: una aglomeración productiva entre Lípez y San Pedro de Atacama. Boletín del Museo Chileno de Arte Precolombino, 22(1), 51-69.

Bray, T. (2004). La alfarería imperial Inka: una comparación entre la cerámica estatal del área de Cuzco y la cerámica de las Provincias. Chungara, 36(2), 365-374.

Coloca, F. (2017a). La arquitectura de Abra de Minas, un sitio con evidencias tardías/incaicas en la puna de Salta, Argentina. Intersecciones en Antropología, 18(2), 245-256.

Coloca, F. (2017b). Interacciones sociales, prácticas rituales y procesos de cambio durante los Períodos Tardío e Inca (ca. 900-1536 DC) en la cuenca de Ratones, Puna de Salta. Tesis doctoral inédita. Facultad de Filosofía y Letras, Universidad de Buenos Aires.

Cruz, P. (2013). De wak'as, minas y jurisdicciones. Apuntes metodológicos en torno a la territorialidad en tiempos del Inka. En A. Presta (Ed.), Aportes multidisciplinarios al estudio de los colectivos étnicos surandinos. Qaraqara-Charka Reflexiones a tres años después (pp. 293-330). Perú: Instituto Francés de Estudios Andinos.

Figueroa, V., Salazar, D., Salinas, H., Núñez Regueiro, P. y Manríquez, G. (2013). Pre-hispanic mining ergology of Northern Chile: an archaeological perspective. Chungara, 45(1), 61-81.

González, C. y Westfall, C. (2008). Atacameños en El Salvador: Nuevas apreciaciones sobre un fardo funerario del Cementerio Las Turquesas. Estudios Atacameños, 35, 49-73.

González, C., Westfall, C. y Castells, C. (2017). Mina Las Turquesas: Lapidaria, secuencia alfarera prehispánica e interrelaciones culturales en un espacio intermodal del Desierto de Atacama, Chile. Estudios Atacameños, 56, 225-251.

García-Albarido Guede, F. (2007). Umiñas en Atacama: aproximación a la lapidaria durante el Periodo Intermedio Tardío y Tawantinsuyu. Informe de Práctica Profesional. Departamento de Antropología, Facultad de Ciencias Sociales,
Universidad de Chile.

Giovannetti, M. (2015). Fiestas y Ritos inka en el Shincal de Quimivil. Buenos Aires: Editorial Punto de Encuentro.

López, G. y Coloca, F. (2015). El sitio Abra de Minas: nuevos aportes para la caracterización de las ocupaciones Tardío/Incas en las tierras altas del Noroeste argentino. Bulletin de I'Institut Français d'Études Andines, 44(1), 141-149.

López, G., Coloca, F., Araya, S., Orsi, J. y Seguí, S. (2015). El sitio Cueva Inca Viejo, salar de Ratones, Puna de Salta: Evidencia arqueológica y procesos de interacción macro-regional. Relaciones de la Sociedad Argentina de Antropología, 40(1), 45-71.

López, G., Coloca, F., Rosenbusch, M. y Solá, P. (2018a). Mining, macro-regional interaction and ritual practices in the SouthCentral Andes: the first evidence for turquoise exploitation from the late prehispanic and inca periods in north-western Argentina (Cueva Inca Viejo, puna of Salta). Journal of Archaeological Science Reports, 17, 81-92

López, G., Coloca, F., Araya, S., Orsi, J., Seguí, S., Rosenbusch, M. y Solá, P. (2018b). Ocupación incaica en Cueva Inca Viejo y Abra de Minas, Puna de Salta, Argentina: minería de turquesa y prácticas rituales. Estudios Atacameños, En prensa.

Núñez, L. (1999). Valoración minero-metalúrgica circumpuneña: menas y mineros para el Inka rey. Estudios Atacameños, 18, 177-222.

Nuñez, L., Agüero, C., Cases, B. y De Souza, P. (2003). El campamento minero Chuquicamata 2 y la explotación cuprífera prehispánica en el desierto de Atacama. Estudios Atacameños, 25, 7- 34

Raffino, R. (1981). Los Inkas del Kollasuyu. La Plata: Ramos.

Salazar, D. (2008). La organización de la producción minera en San José del Abra durante el Período tardío atacameño. Estudios Atacameños, 36, 43-72.

Salazar, D. y Vilches, F. (2014). La arqueología de la minería en el centro-sur andino: Balance y perspectivas. Estudios Atacameños, 48, 5-21.

Salazar, D., Berenguer, J. y Vega, G. (2013). Paisajes minerometalúrgicos inkaicos en Atacama y el altiplano sur de Tarapacá (norte de Chile). Chungara, 45(1), 83-103.

Soto C. y Salazar, D. (2016). Mullu (Spondylussp.) en el Complejo Minero San José del Abra (Alto Loa, Norte de Chile). Intersecciones en Antropología, 17, 129-135.

Westfall, C. y González, C. (2010). Mina Las Turquesas: un asentamiento minero lapidario preincaico en el extremo meridional del área circumpuneña, Región de Atacama, Chile. En Actas del XVII Congreso Nacional de Arqueología Chilena (Volumen 2) (pp. 1073-1083). Valdivia.

Williams, V., Villegas, M., Gheggi, M. y Chaparro, M. (2005). Hospitalidad e intercambio en los valles mesotermales del Noroeste Argentino. Boletín de la PUCP, 9, 335-373. 